\title{
Struma ovarii metastásico, reporte de un caso
}

\author{
Iván Mélendez-García, Moisés Salamanca-García*, Marvin J. Benavides-Maruri y Ma. Edith Salgado-Alday
}

Departamento de Anatomía Patológica, Centro Médico Nacional 20 de Noviembre, Instituto de Seguridad y Servicios Sociales de los Trabajadores del Estado, Ciudad de México, México

\section{Resumen}

Introducción: El struma ovarii comprende el 0.3\% de las neoplasias ováricas. Presentación de caso: Mujer de 46 años con antecedente de struma ovarii. Acude por presentar lesiones hepáticas, resultado de biopsia con folículos tiroideos sin atipia, rodeados de hepatocitos sin atipia, diagnóstico corroborado por inmunohistoquímica. Discusión: El struma ovarii es en la mayoría de las ocasiones un tumor benigno y cuando se realiza el diagnóstico de struma ovarii maligno es controvertido; se ha defendido que el diagnóstico de malignidad siga los criterios histológicos del de tiroides, sin embargo, se ha propuesto que un comportamiento metastásico se considere maligno.

Palabras clave: Struma ovarii. Metástasis. Inmunohistoquímica.

\section{Metastatic struma ovarii, case report}

\section{Abstract}

Background: struma ovarii, comprises $0.3 \%$ of ovarian neoplasms. Clinical case presentation: 46 year old female with a history of struma ovarii. She comes for presenting liver lesions, biopsy results with thyroid follicles without atypia, surrounded by hepatocytes without atypia, diagnosis corroborated by immunohistochemistry. Discussion: struma ovarii, is mostly a beningo tumor, however, diagnosis of malignant struma ovarii is controversial, it has been argued that the diagnosis of malignancy follows the thyroid histological criteria, however, it has been proposed that metastatic behavior is considered malignant.

Key words: Struma ovarii. Metastasis. Immunohistochemistry.

\section{Introducción}

Dentro de la clasificación de teratomas, el struma ovarii, descrito por von KIden en 1895 y en 1899 por Gottschalk, es un tumor raro perteneciente a los tumores de ovario de células germinales. Los tumores de células germinales representan el $20 \%$ de las neoplasias de ovario y de ellos los teratomas representan el $95 \%$. El struma ovarii comprende del 1.4 al $3 \%$ de los teratomas y el $0.3 \%$ de todas las neoplasias ováricas, mientras que el struma ovarii maligno con metástasis se ha visto en menos del $5-6 \%$ de los casos $^{1-7}$.
Del 15 al 20\% de los teratomas ováricos contienen tejido tiroideo, y de estos solo el $5 \%$ se consideran struma ovarii, ya que esta definición se utiliza cuando el tejido tiroideo constituye más del $50 \%$ del teratoma, o cuando contiene menos del $15 \%$ de tejido tiroideo y este es funcional, o cuando presenta algún componente tiroideo maligno $0^{1,4-6}$.

Se presenta un caso de struma ovarii metastásico reportado por el Servicio de Anatomía Patológica del Centro Médico Nacional 20 de Noviembre, Ciudad de México, por el interés que supone debido a su baja incidencia, con una posterior revisión de la literatura sobre las características clínicas y anatomopatológicas de esta entidad.

\footnotetext{
Correspondencia:

*Moisés Salamanca-García

E-mail: patolomsg@yahoo.com.mx

Fecha de recepción: 21-02-2020

Fecha de aceptación: 01-03-2021 DOI: 10.24875/REMQ.20000002

Disponible en internet: 04-06-2021

Rev Esp Méd Quir. 2020;25:111-4 www.remq-issste.com

1665-7330 / @ 2021 Revista de Especialidades Médico-Quirúrgicas. Publicado por Permanyer México SA de CV. Este es un artículo Open Access bajo la licencia CC BY-NC-ND (http://creativecommons.org/licenses/by-nc-nd/4.0/).
} 


\section{Presentación de caso}

Mujer de 46 años es referida al Centro Médico Nacional 20 de Noviembre del Instituto de Seguridad y Servicios Sociales de los Trabajadores del Estado (ISSSTE), para su manejo por probable tumor metastásico. Cuenta con antecedente de struma ovarii funcional hace ocho años, que se trató con cirugía (etapificadora de ovario) y quimioterapia (cuatro ciclos de paclitaxel y cisplatino); posteriormente, por persistencia de aumento de tiroglobulina se realizó tiroidectomía total y se administró iodo radiactivo, con reporte histopatológico de tiroides sin alteraciones histológicas. Entre sus estudios de extensión se encuentra una lesión hepática de características primordialmente quísticas, la cual se refriere que se puncionó en diversas ocasiones, con reportes negativos de malignidad.

A su valoración se encontró clínicamente estable, con distensión abdominal a expensas de tumoración hepática, sin compromiso cardiorrespiratorio, así como un hipotiroidismo secundario con tratamiento sustitutivo; dentro de los estudios de imagen realizados (tomografía axial computarizada, tomografía por emisión de positrones y resonancia magnética) se evidencia una lesión hepática quística de $30 \mathrm{~cm}$ en lóbulo derecho con extensión a lóbulo izquierdo y aparente infiltración a peritoneo. Estudios de laboratorio con reporte de elevación del marcador tumoral Ca-125 en $61.9 \mathrm{U} / \mathrm{ml}$, hemoglobina de $11.6 \mathrm{~g} / \mathrm{dl}$ y cifras de hormona estimulante de la tiroides 10-1, tiroxina total 23.2, tiroxina libre 3.8 y triyodotironina total 3.68 .

La paciente es llevada a colocación de catéter para drenaje hepático, con la obtención de 2,000 ml, que se envía al servicio de anatomía patológica, con reporte citológico de: "contenido de quiste constituido por eritrocitos, no se observan células epiteliales". Posteriormente se realiza resección de tumor hepático, con los siguientes hallazgos quirúrgicos: tumor hepático dependiente de lóbulo derecho, múltiples adherencias a epiplón, pared abdominal y asas intestinales, lóbulo izquierdo con 15 metástasis; por lo cual se marsupializó la apertura de pared quística, con un drenaje de 3,000 ml de contenido de quiste, y se tomó biopsia hepática de lóbulo izquierdo de segmento III.

Dentro del análisis del material recibido en el servicio de anatomía patológica, el diagnóstico correspondiente al material de contenido de quiste se reportó como: «presencia de polimorfonucleares inmersos en un fondo proteináceo». La biopsia referida como «óbulo hepático» se

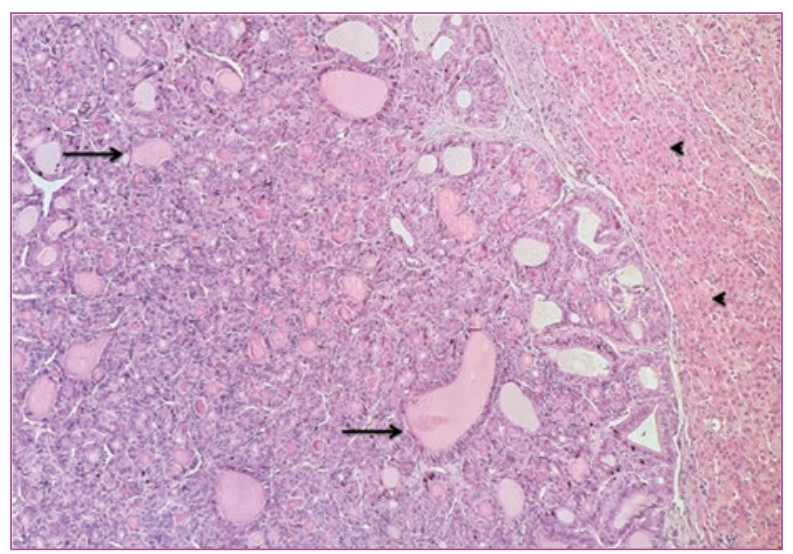

Figura 1. Tejido tiroideo con presencia de folículos de diversos tamaños (flechas), así cómo parénquima hepático adyacente a la lesión (cabezas de flechas) (hematoxilina y eosina, aumento $10 \mathrm{x}$ ).

fijó en formol al 10\%; se trató de un espécimen de $5 \mathrm{x}$ $4.5 \times 1.7 \mathrm{~cm}$, de bordes irregulares, superficie lisa, color café oscuro, opaco, al corte del tejido con presencia de lesiones ovoides, la mayor de $2.5 \mathrm{~cm}$ y la menor de $2 \mathrm{~cm}$ de eje mayor, ambas de color café y consistencia blanda. Al estudio microscópico se observaron lóbulos de parénquima tiroideo separados por septos fibrosos y presencia de folículos tiroideos de diversos tamaños (Fig. 1), los cuales se encontraban revestidos por un epitelio simple cúbico correspondiente a células foliculares sin atipia nuclear, con coloide en su interior (Fig. 2); alrededor de la lesión, presencia de hepatocitos en una disposición de cordones, sin atipia nuclear, con dilatación sinusoidal, así como venas centrolobulillares y espacios porta sin alteraciones histológicas (Fig. 3). Se realiza estudio de inmunohistoquímica con diversos anticuerpos para corroborar el origen de las células y descartar algún otro proceso neoplásico, siendo la vimentina, la inhibina y el específico de hepatocito (HepPar1) negativos en células neoplásicas (Fig. 4), mientras que se presentó inmunorreacción en células neoplásicas con el factor de transcripción tiroidea-1 (Fig. 5), por lo cual el diagnóstico emitido fue: «óbulo hepático con metástasis de struma ovarii».

Días después se da de alta a la paciente por mejoría y por solicitud de esta, y se mantiene en vigilancia por médicos tratantes.

\section{Discusión}

El struma ovarii es en la mayoría de los casos un tumor benigno y cuando se realiza el diagnóstico de 


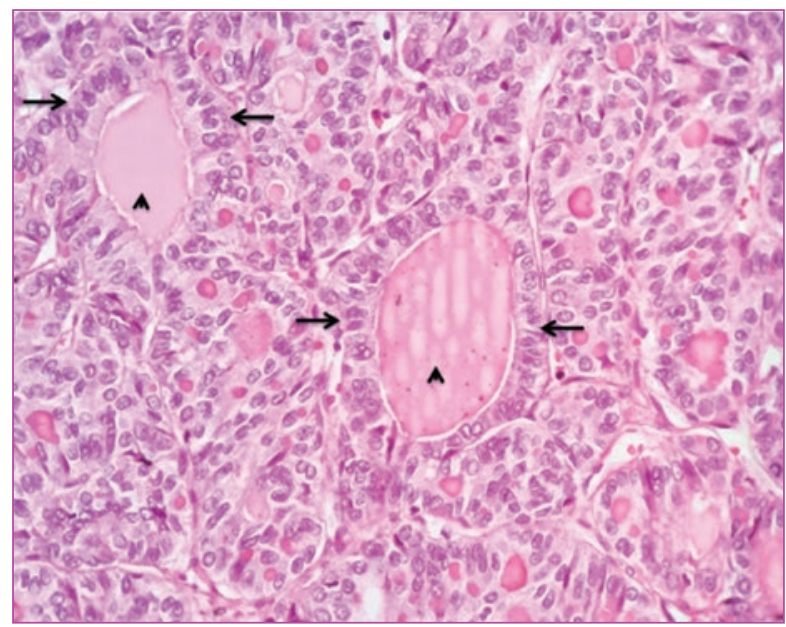

Figura 2. Folículos tiroideos, rodeados por células foliculares (flechas), con material coloide en su interior (cabezas de flecha) (hematoxilina y eosina, aumento $40 \mathrm{x}$ ).

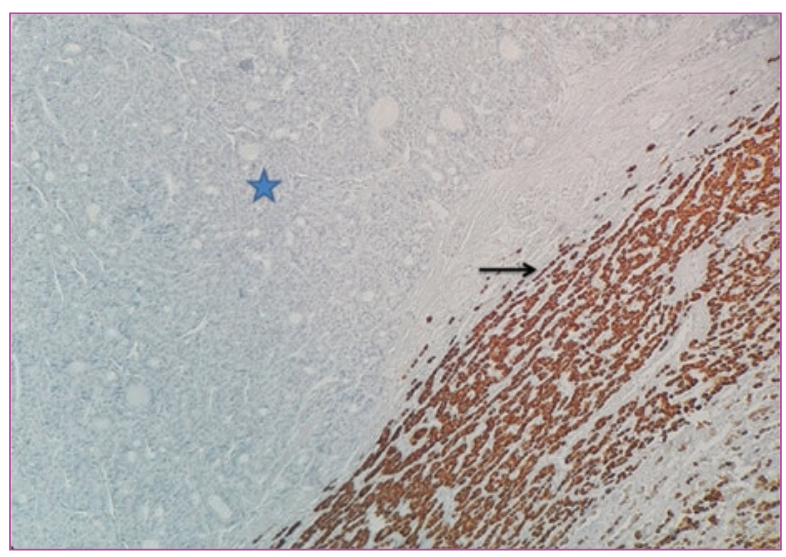

Figura 4. Anticuerpo de específico de hepatocito (HepPar1), el cual resultó negativo en células neoplásicas (estrella) y positivo de manera normal en células hepáticas (flecha) (aumento $10 \mathrm{x}$ ).

struma ovarii maligno, este es controvertido. El diagnóstico histopatológico de malignidad se establece según los criterios para los tumores de la glándula tiroides e incluye la presencia de formaciones papilares revestidas con núcleos de vidrio esmerilado, barras intranucleares, invasión vascular, cuerpos de psamoma y mitosis. La neoplasia maligna histológica en el struma no indica necesariamente una neoplasia maligna biológica. Robboy, et al. clasificaron los tumores en neoplasias malignas biológicas e histológicas. Una neoplasia maligna biológica se definió como: a) tumor ovárico con metástasis más allá del ovario o penetrado en la superficie ovárica, o b) el tumor ovárico ha recurrido. De los 88 casos que estudiaron, 27 se clasificaron como biológicamente malignos, de los cuales 12 casos también se

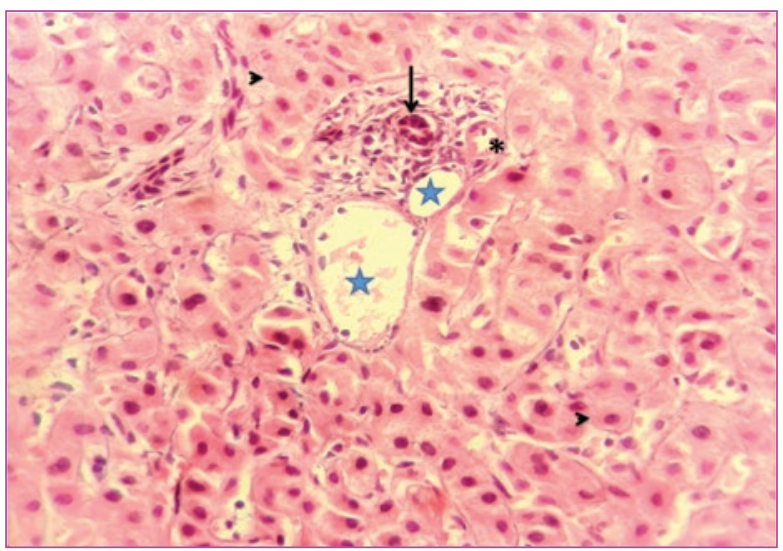

Figura 3. Parénquima hepático adyacente a lesión con hepatocitos (cabeza de flecha), y tríada portal conformada por vena porta (estrella), arteria hepática (asterisco) y conductos biliares (flecha) (hematoxilina y eosina, aumento $40 \mathrm{x}$ ).

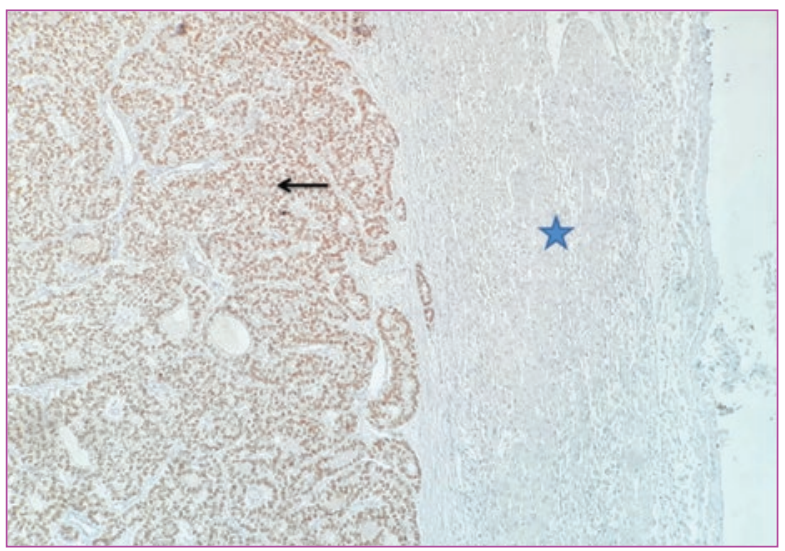

Figura 5. Anticuerpo de factor de transcripción tiroidea-1, el cual resultó positivo en células neoplásicas (flecha) y negativo de manera normal en células hepáticas (estrella) (aumento $10 \mathrm{x}$ ).

clasificaron como histológicamente malignos; el tejido de los 15 casos restantes se identificó como tejido tiroideo benigno. Mientras que de 28 casos que se clasificaron como histológicamente malignos, solo $10 \mathrm{se}$ identificaron como biológicamente malignos. Por lo tanto, la malignidad histológica no se pudo utilizar de manera efectiva para predecir el curso clínico posterior ${ }^{1,6-10}$.

Diversos autores han defendido que el diagnóstico patológico del struma ovarii maligno siga los criterios histológicos de los carcinomas de tiroides, sin embargo, también se ha propuesto que el struma ovarii con comportamiento metastásico se considere maligno ${ }^{11-14}$.

El carcinoma de tiroides que surge en el struma ovarii es más probable que ocurra en lesiones de más de $16 \mathrm{~cm}$, siendo el tipo histológico más común el 
carcinoma papilar de tiroides (44\%), seguido por el carcinoma folicular (30\%), el cual se ha visto que es el tipo con el mayor número de casos con metástasis, y por último la variante folicular del carcinoma papilar (26\%). Los sitios predominantes de metástasis son: estructuras pélvicas (incluido el ovario contralateral), pulmón, hueso, hígado y cerebro, pero la metástasis es rara en pacientes con struma ovarii maligno. Se han encontrado mutaciones de los genes KRAS y BRAF en pacientes con struma ovarii maligno, así como au-

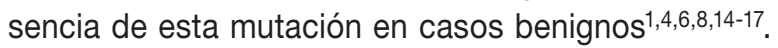

La mayoría de estos tumores suelen ser unilaterales. Las variaciones histológicas del struma benigno incluyen tipos diferenciados como el patrón difuso (incluidos los patrones microfolicular y macrofolicular), los cuales conservan una histología muy semejante a la tiroidea, y los tipos desdiferenciados como el patrón trabecular, pseudotubular y pequeños grupos de células tumorales ${ }^{2,17}$.

Las tasas de supervivencia reportadas del struma ovarii maligno a los 5, 10 y 20 años son del $96.7,94.3$ y $84.9 \%$ respectivamente. No existe un consenso sobre el tratamiento correcto de esta patología debido a la rareza del tumor. Existen informes que han propuesto una estadificación quirúrgica completa para el cáncer de ovario, algunos otros indican que una tiroidectomía total y iodo radiactivo pueden ayudar a la eliminación del tejido tiroideo residual después de la extirpación quirúrgica del tumor primario, así como en casos en que el tumor mida más de $2 \mathrm{~cm}$, presente extensión extraovárica, metástasis a distancia, recurrencia o cáncer de tiroides primario concomitante t,4,6,7,18,19. $^{1}$.

Presentamos el caso de una paciente con antecedente de struma ovarii, la cual presentó ocho años posterior a tratamiento con cirugía etapificadora de ovario, tiroidectomía y iodo radiactivo, metástasis hepáticas con una histología diferenciada, considerado como un caso de neoplasia maligna biológica, con mejoría de la sintomatología posterior a tratamiento quirúrgico del quiste hepático con tumor.

\section{Financiamiento}

Recursos materiales aportados por el Servicio de Anatomía Patológica del Centro Médico Nacional 20 de Noviembre, ISSSTE.

\section{Conflicto de intereses}

Los autores declaran no tener conflicto de intereses.

\section{Responsabilidades éticas}

Protección de personas y animales. Los autores declaran que para esta investigación no se han realizado experimentos en seres humanos ni en animales.

Confidencialidad de los datos. Los autores declaran que han seguido los protocolos de su centro de trabajo sobre la publicación de datos de pacientes.

Derecho a la privacidad y consentimiento informado. Los autores han obtenido el consentimiento informado de los pacientes y/o sujetos referidos en el artículo. Este documento obra en poder del autor de correspondencia.

\section{Bibliografía}

1. Ukita M, Nakai H, Kotani Y, Tobiume T, Koike E, Tsuji I, et al. Long-term survival in metastatic malignant struma ovarii treated with oral chemotherapy: A case report. Oncol Lett. 2014;8(6):2458-62.

2. Cabezas-Palacios MN, Rodríguez-Zarco E, Rodríguez-Jiménez, I, Márquez-Maraver F. Teratoma ovárico maduro e inmaduro, a propósito de un caso. Ginecol Obstet Méx. 2017;85(5):331-7.

3. Alá PMC, García S, Tames FRE, Cañedo GNA, Villarroel SJC. Características anatomopatológicas del teratoma ovárico procedente de ooforectomías, en el Instituto de Gastroenterológico Boliviano-Japonés. Gac Med Bol. 2013;36(2):86-9.

4. Wu M, Hu F, Huang X, Tan Z, Lei C, Duan D. Extensive peritoneal implant metastases of malignant struma ovarii treated by thyroidectomy and 131 I therapy: A case report. Medicine. 2018;97(51):e13867.

5. Barrios-García L, Ruiz-Cáez K, Mendoza-Suárez L, Gómez-Villa J. Struma ovarii: presentación de caso y revisión de la literatura. Rev Colomb Obstet Ginecol. 2014;65(1):79-84.

6. Zhu Y, Wang C, Zhang GN, Shi Y, Xu SQ, Jia SJ, et al. Papillary thyroid cancer located in malignant struma ovarii with omentum metastasis: a case report and review of the literature. World J Surg Oncol. 2016;14(1):17.

7. Wee JY, Li X, Chern BS, Chua IS. Struma ovarii: management and follow-up of a rare ovarian tumour. Singapore Med J. 2015;56(1):35-9.

8. Der-Wei H, Shan-Yin T, Hon-Man C, Yu-Wen Chend, Yu-Chieh C, PiJung $\mathrm{H}$. A rare case of histologic benign struma ovarii with distant metastasis J Clin Gynecol Obstet. 2014;3(3):108-13.

9. Leite I, Cunha TM, Figueiredo JP, Félix A. Papillary carcinoma arising in struma ovarii versus ovarian metastasis from primary thyroid carcinoma: a case report and review of the literature. J Radiol Case Rep. 2013;7(10):24-33.

10. Robboy SJ, Shaco-Levy R, Peng RY, Snyder MJ, Donahue J, Bentley $\mathrm{RC}$, et al. Malignant struma ovarii: an analysis of 88 cases, including 27 with extraovarian spread. Int J Gynecol Pathol. 2009;28(5):405-22.

11. Yücesoy G, Cakiroglu Y, Muezzinoglu B, Besnili B, Yucesoy I. Malignant struma ovarii: a case report. J Korean Med Sci. 2010;25(2):327-9.

12. Devaney K, Snyder R, Norris HJ, Tavassoli FA. Proliferative and histologically malignant struma ovarii: a clinicopathologic study of 54 cases. Int J Gynecol Pathol. 1993;12(4):333-43.

13. Chan SW, Farrell KE. Metastatic thyroid carcinoma in the presence of struma ovarii. Med J Aust. 2001;175(7):373-4.

14. DeSimone CP, Lele SM, Modesitt SC. Malignant struma ovarii: a case report and analysis of cases reported in the literature with focus on survival and I ${ }^{131}$ therapy. Gynecol Oncol. 2003;89(3):543-8.

15. Tzelepis EG, Barengolts E, Garzon S, Shulan J, Eisenberg Y. Unusual case of malignant struma ovarii and cervical thyroid cancer preceded by ovarian teratoma: Case report and review of the literature. Case Rep Endocrinol. 2019;2019:7964126.

16. Flavin R, Smyth P, Crotty P, Finn S, Cahill S, Denning K, et al. BRAF T1799A mutation occurring in a case of malignant struma ovarii. Int $J$ Surg Pathol. 2007;15(2):116-20.

17. Osakabe M, Fukagawa T, Fukagawa D, Sugimoto R, Uesugi N, Ishida $\mathrm{K}$, et al. Struma ovarii with unique histological features: a case report. Int J Clin Exp Pathol. 2017;10(11):11230-3.

18. Lager CJ, Koenig RJ, Lieberman RW, Avram AM. Rare clinical entity: Metastatic malignant struma ovarii diagnosed during pregnancy Lessons for management. Clin Diabetes Endocrinol. 2018;4:13.

19. Llueca A, Maazouzi Y, Herraiz JL, Medina MC, Piquer D, Segarra B, et al. Treatment and follow-up in an asymptomatic malignant struma ovarii: A case report. Int J Surg Case Rep. 2017;40:113-5. 